\title{
Germinación, tiempo hídrico y análisis isotópico de Vicia villosa Roth. bajo condiciones de estrés hídrico y salino
}

Perissé, P., Arias, C. V. y Nogués Mestres, S.

DOI 10.31047/1668.298x.v38.n2.32790

\begin{abstract}
RESUMEN
Vicia villosa es un recurso forrajero potencial en áreas marginales asociado con su resiembra natural y sus atributos agronómicos. Los objetivos del trabajo fueron registrar y analizar el patrón de absorción de agua, determinar el porcentaje de germinación, estimar los parámetros de tiempo hídrico y establecer la composición isotópica de ${ }^{13} \mathrm{C}$ y ${ }^{15} \mathrm{~N}$ de la plántula cuando Vicia villosa germina en condiciones de estrés hídrico y salino. Los ensayos para determinar el patrón de absorción y la respuesta a la germinación se realizaron según el tratamiento: control (agua destilada) y soluciones con potencial agua $\left(\Psi_{\mathrm{a}}\right)$ : -0,3; -0,6; -0,8; - 1 y-1,2 MPa. Los osmolitos fueron poliethylene glicol (PEG) y cloruro de sodio $(\mathrm{NaCl})$. La composición isotópica de la materia orgánica total (MOT) se determinó en condiciones control, -0,8 y -1 MPa. La absorción de un $125 \%$ de agua desencadenó la germinación. Se estimó que con $\Psi_{a}$ de -0,6 y - - , $8 \mathrm{MPa}$ se alcanzaría un $90 \%$ de germinación en estrés hídrico y salino. Los tratamientos de PEG y $\mathrm{NaCl}$ mostraron un enriquecimiento en ${ }^{13} \mathrm{C}$ y un empobrecimiento en ${ }^{15} \mathrm{~N}$ respecto al control. Los resultados experimentales indican que esta especie es más tolerante a la salinidad que al estrés hídrico.
\end{abstract}

Palabras clave: leguminosa forrajera, sequía, salinidad, semilla, $\delta^{13} \mathrm{C}, \delta^{15} \mathrm{~N}$

Perissé, P., Arias, C. V. and Nogués Mestres, S., 2021. Germination, hydrotime and isotopic analysis of Vicia villosa Roth. under water and salinity stress. Agriscientia 38 (2): 89-101

\section{SUMMARY}

Vicia villosa is a potential forage resource in marginal areas associated with its natural reseeding and agronomic attributes. The objectives of this work were to record and analyze the water absorption pattern, determine the germination percentage, estimate the hydrotime parameters and establish the ${ }^{13} \mathrm{C}$ and ${ }^{15} \mathrm{~N}$ isotopic composition of the seedling, when $V$. villosa germinates in water and salinity stress conditions. The assays to determine the water absorption pattern and the response to germination were carried out according to the treatment: control (distilled water) and the solutions with potential water $\left(\Psi_{\mathrm{a}}\right)$ : -0.3; -0.6; -0.8; 
-1 and -1.2 MPa. The osmolytes were Polyethylene Glycol (PEG) and sodium chloride $(\mathrm{NaCl})$. The isotopic composition of the total organic matter (MOT) was determined under control conditions, -0.8 and $-1 \mathrm{MPa}$. The absorption of $125 \%$ of water triggered the germination. It was estimated that $90 \%$ of germination would be achieved with $\Psi_{\mathrm{a}}$ of -0.6 and $-0.8 \mathrm{MPa}$ in water and salinity stress. PEG and $\mathrm{NaCl}$ treatments showed an enrichment in ${ }^{13} \mathrm{C}$ and a depletion in ${ }^{15} \mathrm{~N}$ with respect to the control. The experimental results lead to the conclusion that this species is more tolerant to salinity than to water stress.

Key Words: forage legume, drought, salinity, seed, $\delta^{13} \mathrm{C}, \delta^{15} \mathrm{~N}$

Perissé, P. (ORCID: 0000-0002-7963-7957) y Arias, C. V. (ORCID: 00000003-3345-030X): Universidad Nacional de Córdoba, Facultad de Ciencias Agropecuarias, Botánica Morfológica, Córdoba, Argentina. Nogués Mestres, S. (ORCID: 0000-0002-1694-0924): Universidad de Barcelona, Facultad de Biología, Biología Evolutiva, Ecología y Ciencias Ambientales. Barcelona, España. Correspondencia a: pperisse@agro.unc.edu.ar

\section{INTRODUCCIÓN}

Entre las leguminosas, el género Vicia comprende, según Bryan y Hughes (2011), aproximadamente entre 160 y 200 especies de importancia económica que son cultivadas como abono verde, forraje o alimento para consumo humano por ejemplo: Vicia faba, $V$. sativa, $V$. hirsuta, $V$. narbonensis, $V$. villosa, entre otras (Gunn, 1981; Marin et al., 1998; Haffani et al., 2017). En Europa Central es común el cultivo de $\checkmark$. villosa conocida con el nombre vulgar de "vicia velluda" (Nadal-Moyano et al., 2004) y en Estados Unidos, con el nombre de "veza invernal", se utiliza en siembras de otoño y presenta un mejor desempeño que $V$. sativa. Su adopción en áreas productivas marginales se asocia con su alto potencial de resiembra natural y sus deseables atributos agronómicos (Renzi et al., 2017).

El proceso de germinación es de fundamental importancia ya que influye directamente en el crecimiento, determinando el éxito en el establecimiento del cultivo (Baskin y Baskin, 2014). Durante la imbibición, el movimiento y la velocidad de entrada de agua depende de las características intrínsecas de la semilla y de la permeabilidad de las cubiertas seminales; que a su vez es influenciada por la morfología, composición y el contenido inicial de agua de la semilla (Copeland y McDonald, 2001). Es ampliamente reconocido que las semillas presentan un patrón de absorción de agua trifásico (Hadas y Russo, 1974; Bewley y Black, 1994; Bradford, 1995) propio de cada especie y que las semillas deben alcanzar un nivel mínimo de hidratación (NMH) para desencadenar la germinación (Hadas, 1977; Perissé et al., 2002; Perissé y Planchuelo, 2004).

La sequía por falta de lluvia o por retraso en la irrigación es uno de los factores limitantes para la producción de un cultivo y es un problema severo en muchas regiones del mundo (Lefi et al., 2004). En suelos salinos, sin bien el potencial osmótico puede ser de una magnitud tal que afecte la imbibición de agua, en la mayoría de los casos es el potencial mátrico el que determina la disponibilidad de agua en el suelo para la semilla (Finch-Savage y Bassel, 2016). Además, la salinidad puede tener efectos tóxicos sobre la semilla (Munns, 2002).

La germinación en condiciones limitantes de agua se correlaciona con el alcance del nivel mínimo de hidratación (NMH), que permite iniciar el alargamiento celular y la aparición de la radícula (Hegarty, 1978). Por lo tanto, es necesario conocer si ocurren variaciones en el patrón de absorción cuando las semillas de $V$. villosa se encuentran en medios con diferentes potenciales agua debido a condiciones de estrés hídrico o salino. Sin embargo, aun en un suelo con una adecuada disponibilidad de agua, existe una dispersión entre la primera y la última semilla que completa la emergencia, siendo ésta un indicador de la uniformidad y la calidad de la población de las semillas (Bradford, 1995). El análisis de tiempo hídrico proporciona varios índices de calidad de las semillas en relación con la tolerancia al estrés, la velocidad y la uniformidad de la germinación (Bradford y Still, 2004). Existen varios estudios realizados con esta metodología para evaluar el comportamiento de diferentes 
especies bajo deficiencia hídrica (Hu et al., 2013; Hu et al., 2015; Farahinia et al., 2017; Renzi et al., 2017; Zhang et al., 2020) y bajo condiciones de salinidad (Cavallaro et al., 2016); sin embargo, no se dispone de antecedentes sobre modelo de tiempo hídrico para la germinación de $V$. villosa en condiciones de salinidad.

El estudio de las respuestas fisiológicas de las plantas que crecen bajo condiciones limitantes puede proporcionar información para predecir futuras respuestas en un ambiente cambiante (Taiz y Zeiger, 2010). La identificación de nuevos criterios fisiológicos, como la composición isotópica de la materia orgánica $(\mathrm{MO})$ de las plantas, resulta una herramienta de utilidad para evaluar el impacto del ambiente y la tolerancia de las plantas al estrés (Araus et al., 2008). La abundancia natural de isótopos estables en la MO de las plantas, como el carbono $\left({ }^{13} \mathrm{C}\right)$ y el nitrógeno $\left({ }^{15} \mathrm{~N}\right)$, varía en condiciones de estrés hídrico o salino. Bajo estas condiciones ambientales el flujo de entrada de $\mathrm{CO}_{2}$ en las hojas disminuye por la menor conductancia estomática y esto provoca un enriquecimiento de ${ }^{13} \mathrm{C}$ por la menor discriminación de la enzima Rubisco durante la fotosíntesis (Barbour y Farquhar, 2000). A su vez, estas limitaciones en el mecanismo fotosintético provocan un empobrecimiento de ${ }^{15} \mathrm{~N}$ en la MO (Tcherkez y Hodges, 2008) ya que existe una relación directa entre la fotosíntesis y el metabolismo del nitrógeno (Taiz y Zeiger, 2010). Por lo tanto, se considera a la composición de isótopos de carbono $\left(\delta^{13} \mathrm{C}\right)$ y de nitrógeno $\left(\delta^{15} \mathrm{~N}\right)$ como indicadores para medir los efectos de la sequía y salinidad en las plantas (Serret et al., 2020).

Con esta premisa y con la finalidad de seleccionar especies y cultivares para un ambiente donde predomina el estrés hídrico y salino, se consideran de importancia los estudios sobre esta especie con promisorias posibilidades de implantación para zonas marginales. Los objetivos del presente trabajo fueron: registrar y analizar el patrón de absorción de agua durante la imbibición, determinar el porcentaje de germinación, estimar los parámetros de tiempo hídrico y establecer la composición isotópica de ${ }^{13} \mathrm{C}$ y ${ }^{15} \mathrm{~N}$ de la plántula, cuando $V$. villosa cv. 'Tolse FCA' germina en condiciones de estrés hídrico y salino.

\section{MATERIALES Y MÉTODOS}

\section{Material vegetal}

Las semillas de $V$. villosa Roth. cv. 'Tolse FCA' se obtuvieron de un ensayo experimental realizado en el campo de la Facultad de Ciencias
Agropecuarias, Universidad Nacional de Córdoba, Argentina ( $\left.31^{\circ} 28^{\prime} 44,4^{\prime \prime} \mathrm{S}, 64^{\circ} 00^{\prime} 20,5^{\prime \prime} \mathrm{O}\right)$. Los frutos fueron cosechados y trillados a mano. Las semillas se almacenaron a $4{ }^{\circ} \mathrm{C}$ con un contenido de humedad de 9,6\% hasta el momento de la realización de los ensayos.

\section{Patrón de absorción de agua}

Para determinar el patrón de absorción de agua se colocaron cuatro repeticiones de diez semillas, previamente pesadas en cajas de plástico de $15 \times 15 \times 9 \mathrm{~cm}$, en cuya base se colocó papel de germinación embebido en distintas soluciones, según el tratamiento: control (agua destilada) y diferentes potenciales agua $\left(\Psi_{\mathrm{a}}\right)$ : -0,3; -0,6; -0,8; -1 y-1,2 Mega Pascal (MPa). El osmolito utilizado para simular el estrés hídrico fue polietilenglicol (PEG 6000), los $\Psi_{\text {a }}$ se obtuvieron a partir de distintas concentraciones de PEG, según la fórmula de Michel (1983). Para simular las condiciones de estrés salino se utilizaron soluciones de cloruro de sodio $(\mathrm{NaCl})$ según la metodología de Sosa et al. (2005). Los $\Psi_{\text {a }}$ se controlaron con un microvoltímetro de punto de rocío, marca Wescor (EE UU).

Se aplicó un pre tratamiento de enfriamiento a $5{ }^{\circ} \mathrm{C}$ durante 7 días (International Seed Testing Association [ISTA], 2004). En los tratamientos con PEG, como es un carbohidrato cuya dilución se ve afectada por la temperatura, se prepararon las soluciones correspondientes para el período de $5{ }^{\circ} \mathrm{C}$ y otras para el período en cámara de germinación a $20^{\circ} \mathrm{C}$. Las cajas de germinación se taparon y colocaron en bolsas plásticas cerradas para evitar diferencias de $\Psi_{\text {a }}$ por evaporación y exclusión, y el papel de germinación se renovó con soluciones frescas de PEG y $\mathrm{NaCl}$ cada $72 \mathrm{~h}$ con la finalidad de mantener constante el $\Psi_{\mathrm{a}}$. Luego del pre tratamiento en frío, las cajas se trasladaron a la cámara de germinación a $20 \pm 1^{\circ} \mathrm{C}$ con luz fluorescente fría de 800 a 1200 Lux, con un fotoperíodo de 8/16 h (luz/oscuridad) (ISTA, 2004).

Se determinó el patrón de absorción de agua en función del tiempo, midiendo los gramos de agua absorbida cada 24 h, en relación con la unidad de peso fresco (PF) (Le Deunff et al., 1989; Perissé y Planchuelo, 2004). Se consideró el inicio de la fase 3 cuando se observó la emergencia de $2 \mathrm{~mm}$ de radícula a través de la cubierta seminal (Bewley y Black, 1994).

Se confeccionaron las curvas de absorción de agua en función del tiempo y se determinó el nivel de absorción a partir del cual comienza la fase de crecimiento de la radícula, al que se denominó nivel mínimo de hidratación (NMH). 


\section{Ensayo de germinación}

Las pruebas de germinación se llevaron a cabo según la metodología descripta anteriormente para los siguientes tratamientos: control (agua destilada) y soluciones a $-0,3 ;-0,6 ;-0,8 ;-1$ y -1,2 (MPa), obtenidas con PEG y $\mathrm{NaCl}$ para simular el estrés hídrico o salino respectivamente. Las muestras o unidades experimentales consistieron de 25 semillas con cuatro repeticiones por tratamiento, siguiendo un diseño en bloques completamente aleatorizados. Se realizaron recuentos diarios del número de semillas germinadas, hasta el décimo cuarto día después de la siembra (DDS) en el cual se dio por finalizado el ensayo. La germinación completa se definió como el porcentaje de germinación que es igual al máximo porcentaje obtenido para las semillas que germinan en el tratamiento control (Hadas, 1977).

Se evaluaron las siguientes variables: porcentaje de germinación (PG), porcentaje final de germinación (PFG) y el número de días para alcanzar el 50 \% de germinación (G50).

\section{Parámetros de tiempo hídrico}

Los parámetros de tiempo hídrico se estimaron por medio de los modelos propuestos por Gummerson (1986) y Bradford (1990):

$$
\operatorname{tg}(p)=\frac{\theta_{H}}{\Psi_{a}-\Psi_{B}(p)} \quad \text { Ec. (1) }
$$

donde $\operatorname{tg}(p)$ es el tiempo de germinación para obtener el porcentaje $p$, con el potencial hídrico $\Psi_{\mathrm{a}}$, siendo $\Psi_{\mathrm{B}}(\mathrm{p})$ el potencial de base para un porcentaje de germinación dado y $\theta_{H}$ es la constante de tiempo hídrico.

Asumiendo que los potenciales de base tienen una distribución normal, la mediana de $\Psi_{\mathrm{B}}(50)$ y los valores de desviación estándar $\left(\sigma_{\psi \mathrm{B}}\right)$ se pueden obtener a partir del análisis de regresión probit (Bradford, 1990):

$$
\operatorname{probit}(p)=\frac{\Psi_{a}-\theta_{H} / \operatorname{tg}(p)-\Psi_{B}(50)}{\sigma_{\Psi B}} \quad \text { Ec. (2) }
$$

Para cada tratamiento $i$ se calculó el error cuadrático mediante:

$$
\varepsilon_{i}^{2}=\sum_{J=1}^{n_{i}}\left[p_{g c}(i, j)-p_{g}(i, j)\right]^{2} \text { Ec. }
$$

donde $p_{g c}$ son los porcentajes calculados, $p_{g}$ los porcentajes experimentales, y $n_{i}$ la cantidad de porcentajes en el tratamiento. El error medio se determinó como:

$$
\varepsilon_{\mathrm{mi}}=\sqrt{\frac{\varepsilon_{i}^{2}}{n_{i}}} \quad \text { EC. (4) }
$$

El error cuadrático total para todos los tratamientos se calculó como:

$$
\varepsilon^{2}=\sum_{\mathrm{i}=1}^{\mathrm{n}} \varepsilon_{\mathrm{i}}^{2} \quad \text { EC. (5) }
$$

donde $n$ es la cantidad de tratamientos. El error medio global se determinó como:

$$
\varepsilon_{\mathrm{m}}=\sqrt{\frac{\varepsilon^{2}}{\mathrm{n}}} \text { Ec. }(6)
$$

El coeficiente de regresión $R^{2}$ para cada tratamiento i se calculó como:

$$
R_{i}^{2}=\frac{S_{i}-\varepsilon_{i}^{2}}{S_{i}} \text { EC. (7) }
$$

donde $\varepsilon_{i}^{2}$ es el calculado con la Ec. (3), y $S_{i}$ está dado por

$$
S_{1}=\sum_{i=1}^{n_{i}}\left[p_{g}(i, j)-p_{g m}(i)\right]^{2} \quad \text { Ec. (8) }
$$

Siendo $p_{g m}(i)$ el valor medio del porcentaje en el tratamiento $i$, definido como:

$$
p_{g m}(i)=\frac{1}{n_{i}} \sum_{i=1}^{n_{i}} p_{g}(i, j) \text { Ec. (9) }
$$

El coeficiente de regresión medio para todos los tratamientos se calculó como:

$$
R_{m}^{2}=\frac{1}{n} \sum_{i=1}^{n} R_{i}^{2}
$$

donde $n$ es la cantidad de tratamientos.

\section{Ensayo de recuperación}

Las semillas que no germinaron se sometieron a una prueba de recuperación para indagar si existieron problemas de toxicidad y evaluar la potencialidad de recuperación. Las semillas de cada tratamiento se colocaron a germinar 
en condiciones óptimas (agua destilada, $20^{\circ} \mathrm{C}$ y $8 / 16$ h de luz/oscuridad). A los 7 días se estableció el número de semillas que germinaron denominándolas "semillas recuperadas" (Ungar, 1995).

\section{Composición isotópica de la materia orgánica total (MOT)}

Para el análisis de la composición isotópica de la materia orgánica total (MOT) se seleccionaron cuatro plántulas crecidas bajo condiciones control, - $-0,8$ y $-1 \mathrm{MPa}$ para los tratamientos con PEG y NaCl. Las muestras de MOT se secaron en estufa a $60{ }^{\circ} \mathrm{C}$ durante $48 \mathrm{~h}$, luego se trituraron y homogeneizaron hasta obtener un polvo fino. Se pesaron $0,8 \mathrm{mg}$ de submuestras en cápsulas de estaño (SYLAB Elemental Microanalisys, UK), de $8 \times 5 \mathrm{~mm}$ de tamaño, con microbalanza de precisión (Mettler Toledo, España). Los análisis se realizaron en el Centro Científico y Tecnológico (CCiT) de la Universidad de Barcelona utilizando un Elemental Analyzer Flash 112 (CarboErba, Milán) acoplado a una Interfaz Delta C Conflo III de Espectrometría de Masas de Relación Isotópica (EA-IRMS) (Termo Finnigan, Alemania).

Los resultados de la relación de isótopos de carbono se expresaron con notación $\delta$ y en partes por mil (\%o) de acuerdo con la siguiente ecuación:

$$
\delta^{13} \mathrm{C}(\%)=\frac{R \text { muestra }}{\mathrm{R} \text { estándar }}-1
$$

donde $R$ muestra es la relación ${ }^{13} \mathrm{C} /{ }^{12} \mathrm{C}$ de la MOT y $R$ estándar se refiere a la relación ${ }^{13} \mathrm{C} /{ }^{12} \mathrm{C}$ del estándar internacional V-PDB (Vienna Pee Dee Belemnite) (International Atomic Energy Agency [IAEA], 1983a).

Los resultados de nitrógeno en la MOT también se expresaron en notación $\delta$ utilizando estándares secundarios internacionales de relaciones conocidas ${ }^{15} \mathrm{~N} /{ }^{14} \mathrm{~N} N 1$ (IAEA, 1978a) y $\mathrm{N}_{2}$ sulfato de amonio (IAEA, 1978b) y $\mathrm{NO}_{3}$ nitrato de potasio (IAEA, 1983b) referidas al $N_{2}$ en el aire:

$$
\delta^{15} \mathrm{~N}(\% \circ)=\frac{R \text { muestra }}{\mathrm{R} \text { estándar }}-1
$$

\section{Análisis estadístico}

Para el análisis estadístico de los resultados se utilizó el paquete de software Infostat ( $\mathrm{Di}$ Rienzo et al., 2011). Los efectos de PEG y $\mathrm{NaCl}$ sobre el porcentaje final de germinación (PFG) se analizaron utilizando ANOVA de una vía (con siete niveles de tratamiento: control, $-0,8,-1$, $-1,2 \mathrm{MPa}$ de PEG y NaCl). Los parámetros de la composición isotópica de la MOT se compararon mediante ANOVA de una vía (con cinco niveles de tratamiento: control, $-0,8$ y $-1 \mathrm{MPa}$ de PEG y $\mathrm{NaCl}$ ). Las diferencias entre los valores medios se evaluaron mediante la prueba de Duncan con un nivel de significancia del $5 \%$. Los parámetros medidos fueron graficados utilizando el programa SigmaPlot (versión 11.0).

\section{RESULTADOS Y DISCUSIÓN}

\section{Patrón de absorción de agua}

Durante los primeros tres días de ensayo no se observaron variaciones en el patrón de absorción entre los tratamientos (Figura 1). Sin embargo, las curvas se diferenciaron a partir del cuarto día tanto para los tratamientos con PEG (Figura 1A) como para aquellos con $\mathrm{NaCl}$ (Figura 1B). Si bien la fase I de imbibición quedó claramente delimitada, no ocurrió lo mismo con la fase II, ya que no se observó la meseta correspondiente a la activación metabólica. La absorción de un 125 \% de agua (1250 gr de agua $\mathrm{Kg}^{-1}$ ), desencadenó el paso a la fase III, esto es, la emergencia y el crecimiento
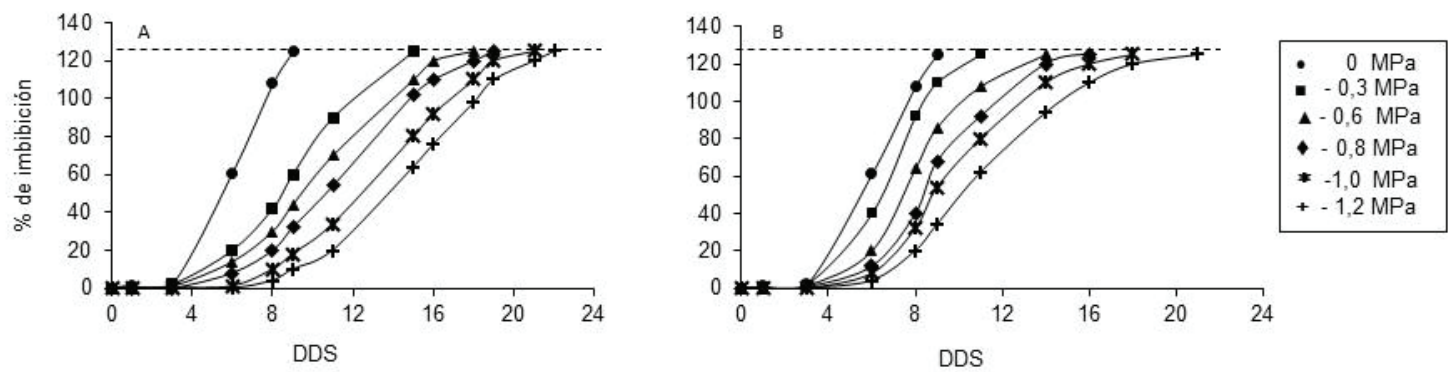

Figura 1. Patrón de absorción de agua en semillas de Vicia villosa cv. 'Tolse FCA' germinadas en condiciones de estrés hídrico, PEG (A) y salino, $\mathrm{NaCl}(\mathrm{B})$ a distintos potenciales agua (MPa). Días después de la siembra (DDS). Línea discontinua: representa el nivel mínimo de hidratación $(\mathrm{NMH})$ 
de la radícula (germinación sensu stricto) y, por lo tanto, determinó el nivel mínimo de hidratación $(\mathrm{NMH})$. Estos datos concuerdan con lo citado para Lupinus albus y Lupinus angustifolius por Perissé et al. (2002), Phaseolus vulgaris por Shafaei et al. (2016) y Cicer arietinum por Gómez-Favela et al. (2017). A su vez, se observó que para un mismo $\Psi_{a}$ los tratamientos con $\mathrm{NaCl}$ alcanzaron el $\mathrm{NMH}$ antes que los tratamientos con PEG.

\section{Ensayo de la germinación}

Los resultados muestran que para el cultivar de $\mathrm{V}$. villosa 'Tolse FCA' bajo la condición control, el $90 \%$ de las semillas germinaron en forma sincrónica a los cuatro días después de la siembra (DDS) y la germinación completa (99\%) se alcanzó a los 12 DDS (Figura 2). Estos resultados constituyen un buen indicador de la uniformidad y por lo tanto de la calidad de la muestra tal como lo señalan Bradford y Still (2004). Además, a -0,3 MPa tanto en los tratamientos con PEG como con $\mathrm{NaCl}$ se alcanzó la germinación completa (Tabla 1).

Sin embargo, se registró una disminución significativa $(p<0,05)$ en el PFG en los distintos tratamientos de estrés con $\Psi_{a}$ menores a -0,3 MPa (Tabla 1). Estos resultados son concordantes con lo señalado para leguminosas como Lupinus albus y Lupinus angustifolius (Perissé et al., 2002)

Tabla 1. Porcentaje final de germinación para $V$. villosa cv. Tolse FCA bajo condiciones de estrés hídrico y salino. Valores medios seguidos de letras iguales no difieren significativamente $(p \geq 0,05)$

\begin{tabular}{lcc}
\hline$\Psi_{a}(\mathrm{MPa})$ & $P E G(\%)$ & $\mathrm{NaCl}(\%)$ \\
\hline 0,0 & $99 \mathrm{a}$ & $99 \mathrm{a}$ \\
$-0,3$ & $99 \mathrm{a}$ & $95 \mathrm{a}$ \\
$-0,6$ & $89 \mathrm{~b}$ & $90 \mathrm{~b}$ \\
$-0,8$ & $61 \mathrm{c}$ & $87 \mathrm{~b}$ \\
$-1,0$ & $33 \mathrm{~d}$ & $80 \mathrm{c}$ \\
$-1,2$ & $15 \mathrm{e}$ & $51 \mathrm{~d}$ \\
\hline
\end{tabular}

y Prosopis strombulífera (Llanes et al., 2005). Bajo condiciones de estrés hídrico, equivalente a -0,6 MPa, el cultivar estudiado reflejó una mejor respuesta a la germinación (PFG 89 \%) comparado con lo reportado por Saglam et al. (2010) para Lens culinaris (PFG 50-70\%) y por Perissé et al. (2002) para L. albus (PFG 75\%) y L. angustifolius (PFG 15\%).

A medida que los potenciales agua disminuyeron, la respuesta a la germinación bajo condiciones de estrés salino fue superior en comparación con las de estrés hídrico (Tabla 1). Esto concuerda con lo señalado por MurilloAmador et al. (2002) para Vigna unguiculata y Cavallaro et al. (2016) para Ceratonia siliqua, quienes reportaron una mejor respuesta a la germinación bajo estrés salino en relación con el estrés hídrico.

Por otro lado, en el control se alcanzó el G50 entre el segundo y tercer DDS y en los tratamientos con PEG y NaCl a $\Psi_{a}$ de hasta -0,8 MPa, se superó el G50 a los diez y cuatro DDS, respectivamente (Figura 2). Las diferencias en relación al tiempo para alcanzar el G50 entre ambos tratamientos, se explican en función de los patrones de absorción de agua, donde a igualdad de potencial, la tasa de absorción de agua fue más lenta en los tratamientos de estrés hídrico.

\section{Parámetros de tiempo hídrico}

La Figura 3 muestra las rectas de tiempo hídrico obtenidas con el modelo de Gummerson (1986). Se observó que para alcanzar el G50 en condiciones de estrés hídrico (PEG), el potencial base $\left(\Psi_{B}\right)$ fue de $-1,00 \mathrm{MPa}$ y para el tratamiento de estrés salino ( $\mathrm{NaCl}$ ) fue de $-1,57 \mathrm{MPa}$. Además, se estimó que con un potencial de -0,6 y -0,8 MPa se alcanzaría un $90 \%$ de germinación bajo condiciones de estrés hídrico y salino, respectivamente. Del mismo modo, fue posible predecir que el tiempo necesario
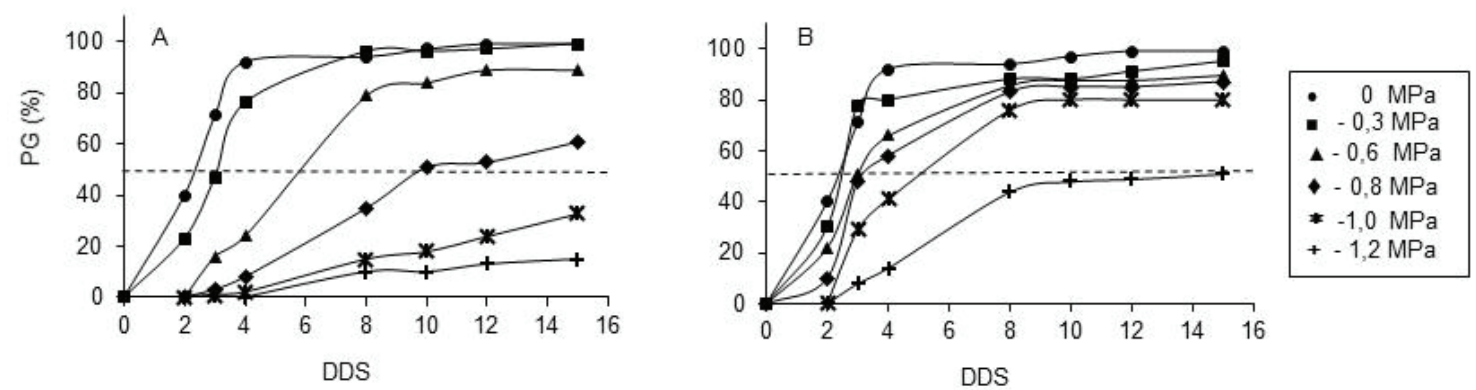

Figura 2. Porcentaje de germinación acumulado de $V$. villosa cv. 'Tolse FCA' en condiciones de estrés hídrico, PEG (A) y salino, NaCl (B) a distintos potenciales agua (MPa). Días después de la siembra (DDS). Línea discontinua: representa el 50 \% de germinación (G50) 
para alcanzar el G50 a un potencial de -0,8 MPa sería de 11 días bajo condiciones de estrés hídrico (Figura 3A) y de cuatro días en salinidad (Figura 3B). Por otra parte, la recta de regresión obtenida del análisis probit (Figura 4) para condiciones de estrés hídrico corta al eje de abscisas en $-1,06 \mathrm{MPa}$, valor correspondiente al $\Psi_{\mathrm{B}}(50)$ (Figura $4 \mathrm{~A}$ ) y para los ensayos de estrés salino, corta al eje de abscisas en -1,54 MPa (Figura 4B).

Los parámetros de tiempo hídrico en ambos modelos se muestran en la Tabla 2; se observó que en condiciones de salinidad el valor de $\theta_{H}$ se incrementó, el $\Psi_{\mathrm{B}}(50)$ disminuyó y el $\sigma_{\psi \mathrm{B}}$ fue mayor comparado con el tratamiento de estrés hídrico.

El $\theta_{H}$ calculado, según el modelo de Gummerson, en estrés hídrico para Vicia villosa (Tabla 2) fue superior en un $24 \%$ al valor medio $(1,78)$ citado por Renzi et al. (2017), y un $69 \%$ al de Vicia amoena $(1,3)$. Sin embargo, fue inferior a los valores para Vicia angustifolia $(3,7)$, Vicia unijuga $(4,3)$ y Vicia sativa $(3,8)$ según Hu et al. $(2015)$. En contraposición, bajo condiciones de salinidad el $\theta_{H}$ para $V$. villosa (Tabla 2) fue inferior a lo señalado por Cavallaro et al. (2016) en ocho genotipos de Ceratonia siliqua $(5,5$ y 9,5). Las diferencias entre los valores de $\theta_{H}$ en las especies estudiadas, si bien son un indicador de la velocidad de germinación, se explican teniendo en cuenta que el valor de la constante $\theta_{H}$ es característico de cada especie

Tabla 2. Parámetros de tiempo hídrico para V. villosa cv. 'Tolse FCA' obtenidos con los modelos de Gummerson y Bradford para los tratamientos de estrés hídrico y salino, y diferencia porcentual entre los resultados de ambos modelos

\begin{tabular}{lcccc}
\hline Tratamiento & Parámetro & Gummerson & Bradford & Dif \% \\
\hline \multirow{3}{*}{ PEG } & $\theta_{H}$ & 2,20 & 2,55 & 16 \\
& $\psi_{B}(50)$ & $-1,00$ & $-1,06$ & 6 \\
& $\sigma_{\psi B}$ & 0,28 & 0,29 & 5 \\
$\mathrm{NaCl}$ & $\theta_{\mathrm{H}}$ & 3,22 & 3,02 & 7 \\
& $\psi_{\mathrm{B}}(50)$ & $-1,57$ & $-1,54$ & 2 \\
& $\sigma_{\psi \mathrm{B}}$ & 0,54 & 0,57 & 6 \\
\hline
\end{tabular}
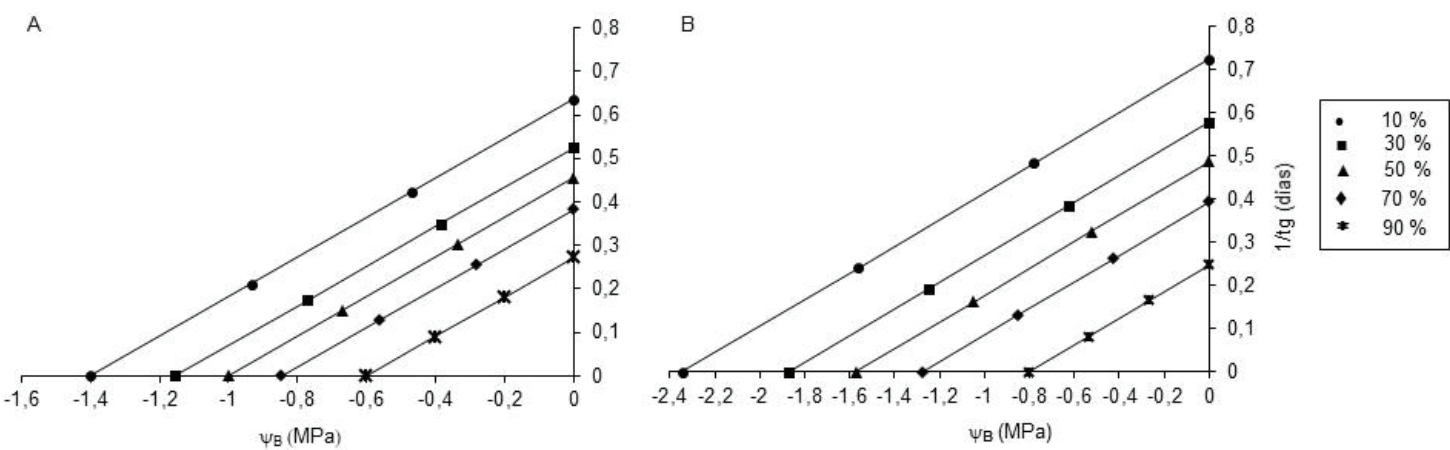

Figura 3. Relación entre potencial base $\left(\Psi_{\mathrm{B}}\right)$, tiempo y porcentaje de germinación de $V$. villosa cV. 'Tolse FCA' bajo condiciones de estrés hídrico, PEG (A) y salino, $\mathrm{NaCl}(\mathrm{B})$ a distintos potenciales agua (MPa), aplicando el modelo de Gummerson $\left(\operatorname{tg}(\mathrm{p})=\frac{\theta_{H}}{\Psi_{\mathrm{a}}-\Psi_{\mathrm{B}}(\mathrm{p})}\right.$ )

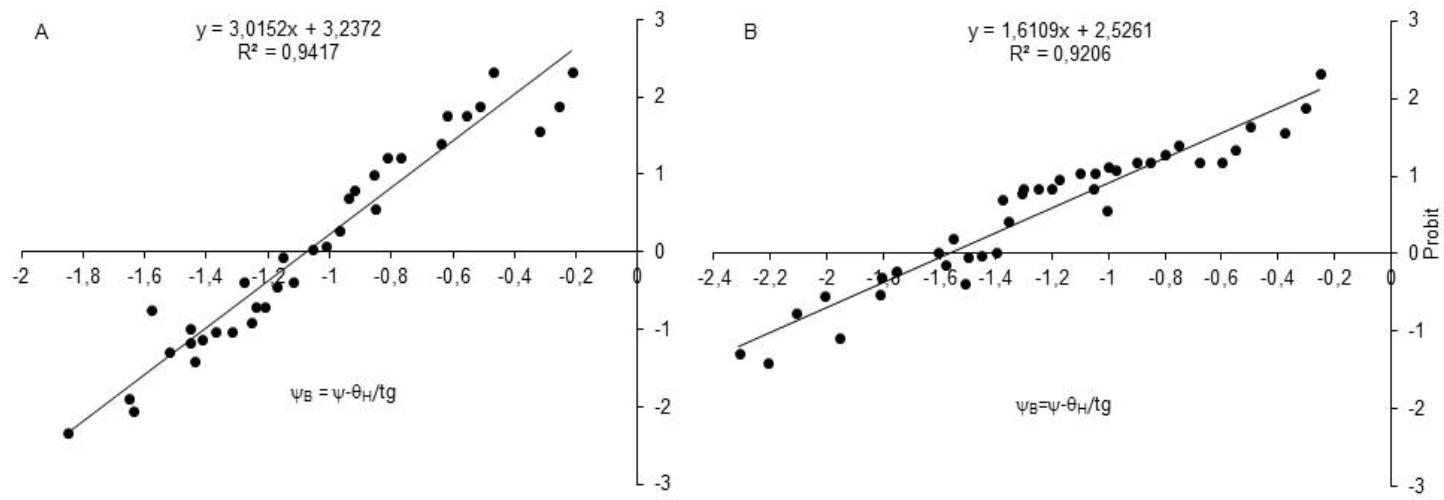

Figura 4. Análisis probit para los distintos niveles de estrés hídrico, PEG (A) y salino, $\mathrm{NaCl}(\mathrm{B})$ a distintos potenciales agua (MPa), aplicando el modelo de Bradford probit $(p)=\frac{\Psi_{2}-\theta_{H} / \operatorname{tg}(p)-\Psi_{B}(50)}{\sigma_{\Psi B}}$ 
(Bradford y Still, 2004).

Por otro lado, el $\Psi_{\mathrm{B}}(50)$ determinado para $V$. villosa en condiciones de estrés hídrico, calculado con el modelo de Gummerson y de Bradford, concordó con lo citado para esta especie $(-1,07)$ por Renzi et al. (2017). Del mismo modo se mantuvo dentro de los rangos señalados por $\mathrm{Hu}$ et al. (2015) para $V$. angustifolia $(-1,29 \mathrm{MPa}), V$. amoena $(-0,64 \mathrm{MPa}), V$. unijuga $(-1,29 \mathrm{MPa})$ y $V$. sativa $(-1,36 \mathrm{MPa})$. También fue consistente con lo indicado por Cavallaro et al. (2016) para distintos genotipos de $C$. siliqua $(-1,28$ y $-1,74 \mathrm{MPa})$ bajo condiciones de salinidad.

La Figura 5 muestra la comparación entre los resultados experimentales y los obtenidos con el modelo de tiempo hídrico a partir de la Ec.1. Las mayores diferencias en el PFG entre los datos experimentales y modelados en los tratamientos con PEG ocurrieron a valores de $\Psi_{\text {a }}-0,6$ y $-0,8 \mathrm{MPa}$, subestimando el modelo entre un 10 y $6 \%$, respectivamente (Figura 5A). Mientras que para los tratamientos de $\mathrm{NaCl}$ los resultados del modelo presentaron diferencias en el PFG inferiores al $10 \%$ con respecto a los experimentales en los tratamientos de hasta $-1 \mathrm{MPa}$ (Figura 5B). Estas diferencias se explican teniendo en cuenta que una imbibición más lenta, a los potenciales mencionados, implicaría una variación en la constante de tiempo hídrico $\left(\theta_{H}\right)$ y pondría de manifiesto las discrepancias a potenciales menores o iguales a -0,6 MPa sin alterar $\Psi_{\mathrm{B}} \circ \sigma_{\mathrm{HB}}$ (Bradford, 1990). Los errores medios globales de los porcentajes de germinación fueron 4,405 y 6,023 y los coeficientes de regresión globales $R^{2}$ fueron 0,97 y 0,96 para los tratamientos con PEG y con $\mathrm{NaCl}$, respectivamente. La Tabla 3 muestra que los errores medios para los tratamientos con PEG son en general inferiores a los correspondientes con los tratamientos con $\mathrm{NaCl}$. Además, los coeficientes de regresión $\mathrm{R}^{2}$ correspondientes a los tratamientos con PEG y con $\mathrm{NaCl}$ muestran un buen ajuste del modelo para ambos tratamientos.

\section{Ensayos de recuperación}

En general, se observó una mayor recuperación de los tratamientos de estrés hídrico (PEG) en comparación con aquellas semillas sometidas a estrés salino (Tabla 4). Los altos porcentajes de recuperación se explicarían por un efecto

Tabla 3. Error medio entre los valores experimentales y modelizados y, coeficiente de regresión para $V$. villosa cv. 'Tolse FCA' bajo condiciones de estrés hídrico (PEG) y salino $(\mathrm{NaCl})$ a distintos potenciales agua (MPa)

\begin{tabular}{lcccc}
\hline & \multicolumn{2}{c}{ PEG } & \multicolumn{2}{c}{$\mathrm{NaCl}$} \\
\hline$\Psi_{a}(\mathrm{MPa})$ & Error medio & $\mathrm{R}^{2}$ & Error medio & $\mathrm{R}^{2}$ \\
\hline 0,0 & 3,76 & 0,9911 & 5,02 & 0,9836 \\
$-0,3$ & 5,21 & 0,9829 & 5,26 & 0,9796 \\
$-0,6$ & 7,84 & 0,96 & 4,03 & 0,9873 \\
$-0,8$ & 2,91 & 0,9875 & 5,91 & 0,9731 \\
$-1,0$ & 2,41 & 0,9662 & 8,39 & 0,9423 \\
$-1,2$ & 1,73 & 0,9321 & 6,58 & 0,9148 \\
\hline
\end{tabular}

Tabla 4. Porcentaje de semillas recuperadas de V. villosa cV. 'Tolse FCA' luego de transferidas a condiciones estándares de germinación según ISTA (2004)

\begin{tabular}{lcc}
\hline$\Psi_{\mathrm{a}}(\mathrm{MPa})$ & $\mathrm{PEG}(\%)$ & $\mathrm{NaCl}(\%)$ \\
\hline$-0,3$ & -- & -- \\
$-0,6$ & 100 & -- \\
$-0,8$ & 82 & 25 \\
$-1,0$ & 92 & 0 \\
$-1,2$ & 89 & 13 \\
\hline
\end{tabular}
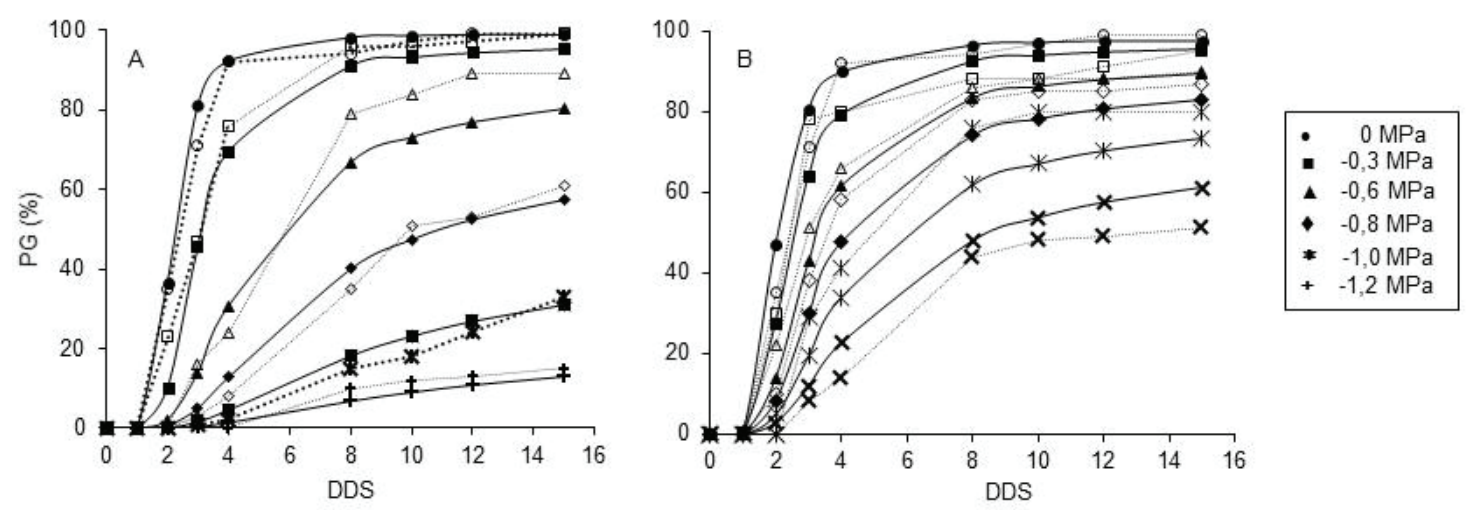

Figura 5. Comparación de los porcentajes de germinación acumulados experimentales y modelizados con el tiempo hídrico para V. villosa cv. 'Tolse FCA' bajo condiciones de estrés hídrico, PEG (A) y salino, NaCl (B) a distintos potenciales agua (MPa). Línea discontinua: datos experimentales, línea continua: datos modelizados. Días después de la siembra (DDS). 
osmoprotector del PEG. Esto es debido a que el sistema de membranas del embrión, que previo a la imbibición de las semillas se encuentran en fase gel, cuando se embeben lentamente restablecen su composición líquido cristalina hasta completar la fase I del patrón de absorción de agua y el embrión se mantiene en la fase II (Bewley y Black, 1994). Así, las membranas conservan su propiedad de permeabilidad selectiva y evitan la pérdida de electrolitos (Bewley y Black, 1994). El embrión mantiene su viabilidad y sólo se evita el pasaje a la fase III. Contrariamente, en los tratamientos con estrés salino las semillas imbiben rápidamente y las membranas en la transición de la fase gel a líquido cristalino conducen al daño por imbibición violenta. Los bajos porcentajes de recuperación, en las semillas no germinadas de los tratamientos más severos de estrés salino, expresan la alteración del equilibrio iónico y acuoso que genera un estrés hiperosmótico, desbalance iónico y toxicidad, que compromete la viabilidad de las semillas expuestas a altas concentraciones salinas (Munns, 2002; Taiz y Zeiger, 2010).

\section{Composición isotópica de la materia orgánica total (MOT)}

La composición isotópica de ${ }^{13} \mathrm{C}\left(\delta^{13} \mathrm{C}\right)$ en la MOT de las plántulas germinadas y crecidas en condiciones control fue cercano al valor promedio para plantas $\mathrm{C}_{3}(-26 \%)$ como lo indican Cerling et al. (1997) (Figura 6A). Las plántulas crecidas en condiciones control presentaron un desarrollo de plántula normal con la presencia de nomofilos fotosintetizantes. Por lo tanto, el menor contenido de ${ }^{13} \mathrm{C}$ fijado en la materia orgánica vegetal se puede relacionar con la discriminación ejercida por la enzima Rubisco, hacia el isótopo más pesado durante el proceso de fotosíntesis de estas hojas (Farquhar et al., 1989).

En las plántulas bajo los tratamientos PEG y $\mathrm{NaCl}$, la $\delta^{13} \mathrm{C}$ de la MOT se mantuvo dentro del rango esperado (-22 a $-30 \%$ ) para plantas $\mathrm{C}_{3}$, como sostienen Cerling et al. (1997), pero se observó un enriquecimiento significativo $(p<0,05)$ en el isótopo más pesado $\left({ }^{13} \mathrm{C}\right)$ en dichos tratamientos con respecto al control (Figura 6A). Estos resultados podrían estar relacionados con la menor disponibilidad de agua en el medio de crecimiento, lo que conduce al cierre de estomas o disminución de la conductancia estomática para reducir la pérdida de agua por transpiración. Cuando el flujo de $\mathrm{CO}_{2}$ se ve reducido la Rubisco discrimina menos al ${ }^{13} \mathrm{C}$ en su incorporación al Ciclo Calvin (Barbour y Farquhar, 2000).
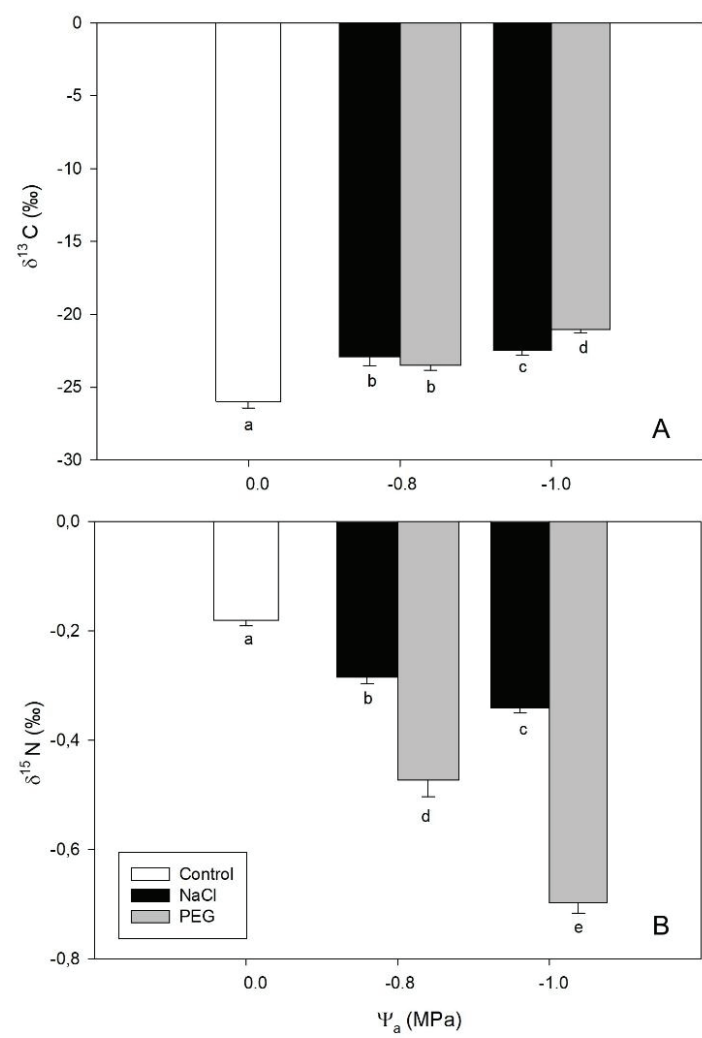

Figura 6. Composición isotópica de carbono 13 (A) y nitrógeno 15 (B) en la materia orgánica total de plántulas de V. villosa cV. 'Tolse FCA' crecidas bajo condiciones de estrés hídrico, PEG y salino, $\mathrm{NaCl}$ a distintos potenciales agua (MPa)

La composición isotópica de ${ }^{15} \mathrm{~N}\left(\delta^{15} \mathrm{~N}\right)$ en la MOT fue de $-0,2 \%$ o para las plántulas crecidas bajo condiciones control. Este resultado estuvo dentro del valor esperado para plantas que dependen únicamente de simbiontes bacterianos para la fijación de $\mathrm{N}_{2}$ ya que el $\delta^{15} \mathrm{~N}$ del aire es cero. En concordancia con lo señalado por Yoneyama et al. (1986) y Makarov (2009), el primer fraccionamiento isotópico ocurre durante la fijación simbiótica de $\mathrm{N}_{2}$ con una preferencia por el ${ }^{14} \mathrm{~N}$ sobre ${ }^{15} \mathrm{~N}$, con un valor $\delta^{15} \mathrm{~N}$ entre $-0,2$ a $-2 \%$ en la MOT de toda la planta. Debido a las condiciones experimentales de crecimiento en este ensayo, las plántulas presentaron una limitación en la formación de nódulos y la fijación de nitrógeno atmosférico $\left(\mathrm{N}_{2}\right)$ por parte de las raíces. Por lo tanto, este valor de la $\delta^{15} \mathrm{~N}$ procedería de la reserva proteica almacenada en la semilla y que fue el $\mathrm{N}_{2}$ fijado por los nódulos bacterianos de la planta madre (Yoneyama et al., 1986). Este resultado es consistente con lo indicado por Piao y Liu (2012), quienes citaron un valor de la $\delta^{15} \mathrm{~N}$ en las semillas de $\mathrm{V}$. villosa de $-0,3 \%$. 
En las plántulas bajo los tratamientos PEG y $\mathrm{NaCl}$ la $\delta^{15} \mathrm{~N}$ en la MOT se mantuvo dentro del rango esperado $(-10 \quad a+10 \%)$ indicados por Evans (2001). Sin embargo, se observó un empobrecimiento significativo $(p<0,05)$ en el isótopo más pesado $\left({ }^{15} \mathrm{~N}\right)$ respecto al control (Figura $6 \mathrm{~B})$. El empobrecimiento de ${ }^{15} \mathrm{~N}$ en la MOT bajo condiciones de estrés podría estar relacionado con las limitaciones de los mecanismos fotosintéticos de la planta en respuesta al medio de crecimiento (Tcherkez y Hodges, 2008).

Como se mencionó anteriormente, la disminución de la conductancia estomática debido al incremento de los $\Psi_{\text {a }}$ ya sea por salinidad 0 sequía, es la principal causa en la disminución del mecanismo fotosintético y crecimiento en la plántula (Munns y Tester, 2008). Las biomoléculas como aminoácidos, proteínas, ácidos nucleicos, coenzimas, fosfolípidos y clorofilas están constituidas por esqueletos carbonados y nitrógeno, lo que evidencia la relación entre la fotosíntesis y el metabolismo del nitrógeno. Además, coexiste una dependencia de ATP y NADPH producido en la fotosíntesis, con las reacciones metabólicas del nitrógeno (Taiz y Zeiger, 2010). Por lo tanto, la alteración en los procesos serían los determinantes de la composición isotópica ${ }^{15} \mathrm{~N}$ en la plántula.

A su vez, se observó que a menor potencial hídrico (-1 $\mathrm{MPa}$ ) hubo un mayor enriquecimiento en ${ }^{13} \mathrm{C}$ y fraccionamiento de ${ }^{15} \mathrm{~N}$, siendo más significativo $(p<0,05)$ en el tratamiento con PEG respecto al tratamiento $\mathrm{NaCl}$. Estos resultados podrían estar asociados a la menor disponibilidad de agua retenida por el osmolito PEG en el medio de crecimiento.

\section{CONCLUSIONES}

El alto porcentaje de germinación (99%) alcanzado por las semillas de Vicia villosa cv. 'Tolse FCA', bajo condiciones control, evidenció una buena calidad y uniformidad de la muestra de semillas.

El estudio reveló que el nivel mínimo de hidratación (NMH) requerido por las semillas para germinar es de $125 \%$ de imbibición de agua sobre el peso fresco y, que existe una variación en la curva de patrón de absorción de agua a medida que disminuyen los potenciales agua, tanto para el tratamiento de estrés hídrico como el salino.

Los resultados experimentales permiten concluir que $V$. villosa cv. 'Tolse FCA' es más tolerante a la salinidad que al estrés hídrico en la germinación. Si bien ambos tratamientos provocaron un retraso en el proceso de germinación y la emergencia, el $\mathrm{NaCl}$ fue menos perjudicial que los tratamientos con PEG. Sin embargo, el potencial de recuperación fue significativamente alto en las semillas luego del estrés hídrico, lo que evidencia que la viabilidad de las semillas se mantiene. Los parámetros de tiempo hídrico y la composición isotópica de ${ }^{13} \mathrm{C}$ y ${ }^{15} \mathrm{~N}$ en la materia orgánica también pusieron de manifestó que esta especie sometida a estrés salino exhibe una mejor respuesta que bajo condiciones de estrés hídrico. Por lo tanto, el cultivar 'Tolse FCA' de $V$. villosa mostraría un potencial promisorio para su uso como pastura o abono verde en suelos salinos o con déficit hídrico.

A su vez, el uso de los parámetros de tiempo hídrico y la composición isotópica de la MO como integradores fisiológicos podrían ser de utilidad en programas de mejoramiento como criterios de selección desde las etapas tempranas de crecimiento y de esta manera identificar cultivares de $V$. villosa con mejor comportamiento y tolerancia al estrés hídrico y salino.

\section{AGRADECIMIENTOS}

Esta investigación contó con la financiación de la Secretaría de Ciencia y Técnica (SECyT) de la Universidad Nacional de Córdoba. Los autores agradecen la colaboración del Ing. Ricardo Luis Marini en la implementación de los modelos matemáticos.

\section{BIBLIOGRAFÍA}

Araus, J. L., Slafer, G. A., Royo, C. y Serret, M. D. (2008). Breeding for yield potential and stress adaptation in cereals. Critical Reviews in Plant Sciences, 27(6), 377412. https://doi.org/10.1080/07352680802467736

Barbour, M. M. y Farquhar, G. D. (2000). Relative humidity and $\mathrm{ABA}$ induced variation in carbon and oxygen isotope ratios of cotton leaves. Plant, Cell \& Environment, 23(5), 473-485. https://doi.org/10.1046/ j.1365-3040.2000.00575.x

Baskin, C. y Baskin, J. (2014). Seeds: Ecology, Biogeography, and Evolution of Dormancy and Germination (2a ed.). Academic Press.

Bewley, J. y Black, M. (1994). Seeds. Physiology of Development and Germination (2a ed.). Plenum Press.

Bradford, K. (1990). A Water Relations Analysis of Seed Germination Rates. Plant Physiology, 94(2), 840-849. https://doi.org/10.1104/pp.94.2.840

Bradford, K. (1995). Water relations in seed germination. En J. Kigel y G. Galili (Eds.), Seed development and 
germination (pp. 351-396). Routledge. https://doi. org/10.1201/9780203740071

Gunn, C. R. (1981). Seeds of Leguminosae. En R. M. Polhill y P. H. Raven (Eds.), Advances in Legume systematic (Parte 2, pp. 913-925). Kew Royal Botanic Gardens.

Bradford, K. y Still, D. (2004). Application of hydrotime analysis in seed testing. Seed Technology, 26, 75-85.

Bryant, J. A. y Hughes, S. G. (2011). Vicia. En C. Kole (Ed.), Wild crop relatives: Genomic and breeding resources legume crops and forage (pp. 273-289). Springer.

Cavallaro, V., Barbera, A. C., Maucieri, C., Gimma, G., Scalisi, C. y Patanè, C. (2016). Evaluation of variability to drought and saline stress through the germination of different ecotypes of carob (Ceratonia siliqua L.) using a hydrotime model. Ecological Engineering, 95, 557566. https://doi.org/10.1016/i.ecoleng.2016.06.040

Cerling, T. E., Harris, J. M., MacFadden, B. J., Leakey, M. G., Quade, J., Eisenmann, V. y Ehleringer, J. R. (1997). Global vegetation change through the Miocene/ Pliocene boundary. Nature, 389, 153-158. https://doi. org/10.1038/38229

Copeland, L. O. y McDonald, M. B. (2001). Principles of seed science and technology (4a ed.). Springer. https://doi.org/10.1007/978-1-4615-1619-4

Di Rienzo, J. A., Casanoves, F., Balzarini, M. G., Gonzalez, L., Tablada, M. y Robledo, C. W. InfoStat (versión 2011) [Software] Córdoba, Argentina: Grupo InfoStat, FCA, Universidad Nacional de Córdoba. http://www. infostat.com.ar.

Evans, R. D. (2001). Physiological mechanisms influencing plant nitrogen isotope composition. Trends in Plant Science, 6(3), 121-126. https://doi. org/10.1016/S1360-1385(01)01889-1

Farahinia, P., Sadat-noori, S. A., Mortazavian, M. M., Soltani, E. y Foghi, B. (2017). Hydrotime model analysis of Trachyspermum ammi (L.) Sprague seed germination. Journal of Applied Research on Medicinal and Aromatic Plants,5, 88-91. https://doi. org/10.1016/i.jarmap.2017.04.004

Farquhar, G. D., Ehleringer, R. y Hubick, K. T. (1989). Carbon Isotope Discrimination and Photosynthesis. Annual Review of Plant Physiology and Plant Molecular Biology, 40, 503-537.

Finch-Savage, W. E. y Bassel, G. W. (2016). Seed vigour and crop establishment: extending performance beyond adaptation. Journal of Experimental Botany, 67(3), 567-591. https://doi.org/10.1093/ixb/erv490

Gómez-Favela, M. A., García-Armenta, E., Reyes-Moreno, C., Garzón-Tiznado, J. A., Perales-Sánchez, J. X. K., Caro-Corrales, J. J. y Gutiérrez-Dorado, R. (2017). Modelling of water absorption in chickpea (Cicer arietinum L.) seeds grown in Mexico's northwest. Revista Mexicana de Ingeniera Quimica, 16, 179-191.

Gummerson, R. J. (1986). The effect of constant temperatures and osmotic potentials on the germination of sugar beet. Journal of Experimental Botany, 37(6), 729-741. https://doi.org/10.1093/ $\mathrm{jxb} / 37.6 .729$

Gunn, C. R. (1981). Seeds of Leguminosae. En R. M. Polhill y P. H. Raven (Eds.), Advances in Legume systematic (Parte 2, pp. 913-925). Kew Royal Botanic Gardens.

Hadas, A. (1977). A Simple Laboratory Approach to Test and Estimate Seed Germination Performance Under Field Conditions. Agronomy Journal, 69(4), 582-588. https://doi.org/10.2134/agronj1977.00021962006900 040015x

Hadas, A., y Russo, D. (1974). Water Uptake by Seeds as Affected by Water Stress, Capillary Conductivity, and Seed Soil Water Contact. II. Analysis of Experimental Data. Agronomy Journal, 66(5), 647-652. https://doi. org/10.2134/agronj1974.00021962006600050013x

Haffani, S., Mezni, M., Ben Nasri, M. y Chaibi, W. (2017). Comparative leaf water relations and anatomical responses of three vetch species (Vicia narbonensis L., V. sativa L. and V. villosa Roth.) to cope with water stress. Crop and Pasture Science, 68(7), 691-702. https://doi.org/10.1071/CP17029

Hegarty, T. W. (1978). The physiology of seed hydration and dehydration, and the relation between water stress and the control of germination: a review. Plant, Cell \& Environment, 1(2), 101-119. https://doi. org/10.1111/j.1365-3040.1978.tb00752.x

Hu, X. W., Fan, Y., Baskin, C. C., Baskin, J. M. y Wang, Y. R. (2015). Comparison of the effects of temperature and water potential on seed germination of Fabaceae species from desert and subalpine Grassland. American Journal of Botany, 102(5), 649-660. https:/l doi.org/10.3732/ajb.1400507

Hu, X. W., Li, T., Wang, J., Wang, Y., Baskin, C. C. y Baskin, J. M. (2013). Seed dormancy in four Tibetan Plateau Vicia species and characterization of physiological changes in response of seeds to environmental factors. Seed Science Research, 23(2), 133-140. https://doi.org/10.1017/S0960258513000019

International Atomic Energy Agency (1983a). Vienna Pee Dee Belemnite (V-PDB). (Standard No. NBS19). https://nucleus.iaea.org/sites/ReferenceMaterials/ Pages/NBS19.aspx

International Atomic Energy Agency (1983b). Potassium nitrate. (Standard No. IAEA NO $\mathrm{N}_{3}$ ). https://nucleus.iaea. org/sites/ReferenceMaterials/Pages/IAEA-NO-3.aspx

International Atomic Energy Agency (1978a). Ammonium sulfate. (Standard No. IAEA N1). https://nucleus.iaea. 
org/sites/ReferenceMaterials/Pages//AEA-N-1.aspx

International Atomic Energy Agency (1978b). Ammonium sulfate. (Standard No. IAEA N $N_{2}$ ). https://nucleus.iaea. org/sites/ReferenceMaterials/Pages/IAEA-N-2.aspx

International Seed Testing Association (ISTA) (2004). International rules for seed testing. https://www. seedtest.org/en/home.html

Le Deunff, Y., Ballot, S. y Toubou, C. (1989). Hydratation des graines de lupin blanc et relargage des électrolytes. Seed Science and Technology, 17, 325340.

Lefi, E., Medrano, H. y Cifre, J. (2004). Water uptake dynamics, photosynthesis and water use efficiency in field-grown Medicago arborea and Medicago citrina under prolonged Mediterranean drought conditions. Annals of Applied Biology, 144(3), 299-307. https:// doi.org/10.1111/j.1744-7348.2004.tb00345.x

Llanes, A., Reinoso, H. y Luna, V. (2005). Germination and Early Growth of Prosopis strombulifera Seedlings in Different Saline Solutions. World Journal of Agricultural Sciences, 1(2), 120-128.

Makarov, M. I. (2009). The nitrogen isotopic composition in soils and plants: Its use in environmental studies (A Review). Eurasian Soil Science, 42, 1335-1347. https://doi.org/10.1134/S1064229309120035

Marin, P. D., Boza, P., Merkukov, L. J., Krsti, B., Petkovi, B. y Velji, M. (1998). Seed sculpturing of selected European Vicia L. species (Fabaceae) and their taxomomical evaluation. Seed Science and Technology, 26, 17-32.

Michel, B. (1983). Evaluation of the water potentials of solutions of polyethylene glycol 8000 both in the absence and presence of other solutes. Plant Physiology, 72, 66-70. https://doi.org/10.1104/ pp.72.1.66

Munns, R. (2002). Comparative physiology of salt and water stress. Plant, Cell \& Environment, 25(2), 239-250. https://doi.org/10.1046/j.0016-8025.2001.00808.x

Munns, R. y Tester, M. (2008). Mechanisms of salinity tolerance. Annual Review of Plant Biology, 59, 651-681. https://doi.org/10.1146/annurev. arplant.59.032607.092911

Murillo-Amador, B., López-Aguilar, R., Kaya, C., Larrinaga-Mayoral, J. y Flores-Hernández, A. (2002). Comparative effects of $\mathrm{NaCl}$ and polyethylene glycol on germination, emergence and seedling growth of cowpea. Journal of Agronomy and Crop Science, 188(4), 235-247. https://doi.org/10.1046/j.1439037X.2002.00563.X

Nadal-Moyano, S., Moreno-Yagüela, M. T. y CuberoAslmeron, J. I. (2004). Las leguminosas grano en la agricultura moderna. Mundi-Prensa

Perissé, P., Aiazzi, M. T. y Planchuelo, A. (2002). Water uptake and germination of Lupinus albus L. and Lupinus angustifolius L. under water stress. Seed Science and Technology, 30(2), 289-298.

Perissé, P. y Planchuelo, A. M. (2004). Seed coat morphology of Lupinus albus L. and Lupinus angustifolius L. in relation to water uptake. Seed Science and Technology, 32, 69-77. https://doi. org/10.15258/sst.2004.32.1.08

Piao, H. y Liu, C. (2012). Response of biomass accumulation and nodulation by Vicia villosa to soil conditions: Evidence from $13 \mathrm{C}$ and $15 \mathrm{~N}$ isotopes. Chinese Journal of Geochemistry, 31, 111-119. https://doi.org/10.1007/s11631-012-0557-3

Renzi, J. P., Chantre, G. R. y Cantamutto, M. A. (2017). Vicia villosa ssp. villosa Roth field emergence model in a semiarid agroecosystem. Grass and Forage Science, 73, 146-158. https://doi.org/10.1111/gfs.12295

Sa lam, S., Day, S., Kaya, G. y Gürbüz, A. (2010). Hydropriming increases germination of lentil (Lens culinaris Medik.) under water stress. Notulae Scientia Biologicae, 2(2), 103-106.

Serret, M. D., Al-Dakheel, A. J., Yousfi, S., FernáandezGallego, J. A., Elouafi, I. A. y Araus, J. L. (2020). Vegetation indices derived from digital images and stable carbon and nitrogen isotope signatures as indicators of date palm performance under salinity. Agricultural Water Management, 230, 105949 https:/l doi.org/10.1016/j.agwat.2019.105949

Shafaei, S. M., Masoumi, A. A. y Roshan, H. (2016). Analysis of water absorption of bean and chickpea during soaking using Peleg model. Journal of the Saudi Society of Agricultural Sciences, 15(2), 135144. https://doi.org/10.1016/j.jssas.2014.08.003

SigmaPlot (versión 11.0) (2008) [Software]. San José, California, EE. UU: Systat Software, Inc.

Sosa, L., Llanes, A., Reinoso, H., Reginato, M. y Luna, V. (2005). Osmotic and Specific Ion Effects on the Germination of Prosopis strombulifera. Annals of Botany, 96(2), 261-267. https://doi.org/10.1093/aob/ $\underline{\operatorname{mci} 173}$

Taiz, L. y Zeiger, E. (2010). Plant Physiology (5a ed.). Sinauer Associates.

Tcherkez, G. y Hodges, M. (2008). How stable isotopes may help to elucidate primary nitrogen metabolism and its interaction with (photo)respiration in C3 leaves. Journal of Experimental Botany, 59(7), 1685-1693. https://doi.org/10.1093/ixb/erm115

Ungar, I. (1995). Seed germination and seed-bank ecology in halophytes. En J. Kigel y G. Galili (Eds.). Seed development and germination (pp. 599-627). Routledge.

Yoneyama, T., Fujita, K., Yoshida, T., Matsumoto, T., Kambayashi, I. y Yazaki, J. (1986). Variation in natural 
abundance of $15 \mathrm{~N}$ among plant parts and in $15 \mathrm{~N} / 14 \mathrm{~N}$ fractionation during N2 fixation in the legume-rhizobia symbiotic system. Plant and Cell Physiology, 27(5), 791-799. https://doi.org/10.1093/oxfordjournals.pcp. $\underline{\mathrm{a} 077165}$
Zhang, R., Luo, K., Chen, D., Baskin, J., Baskin, C., Wang, Y. y Hu, X. (2020). Comparison of Thermal and Hydrotime Requirements for Seed Germination of Seven Stipa Species From Cool and Warm Habitats. Frontiers in Plant Science, 11, 560714. https://doi. org/10.3389/fpls.2020.560714 